\title{
TRANSIENT OPTICAL ABSORPTION SPECTROSCOPY OF THE PHOTOCHEMICAL SPIROPYRAN-MEROCYANINE CONVERSION
}

\author{
Niko P. ERNSTING \\ Abteilung Laserphysik, Max-Planck-Institut für Biophysikalische Chemie, D-3400 Göttingen, Federal Republic of Germany
}

Received 19 April 1989

\begin{abstract}
The photochemical ring-opening reaction of $1^{\prime}, 3^{\prime}, 3^{\prime}$-trimethylspiro-[2H-1-benzopyran-2,2'-indoline $]$ has been followed by transient absorption spectroscopy, covering the range $370-650 \mathrm{~nm}$ with a temporal resolution of 0.42 ps. During the pump pulse, induced transient absorption is observed only at wavelengths shorter than $500 \mathrm{~nm}$, with a maximum near $440 \mathrm{~nm}$. The absorption at $440 \mathrm{~nm}$ decays within $\approx 5 \mathrm{ps}$, while a broad absorption band appears at $550 \mathrm{~nm}$ in the region characteristic of merocyanine absorption. The risetime of the absorption at $550 \mathrm{~nm}$ is approximately $1.3 \mathrm{ps}$. The reaction is described as a barrierless photoisomerisation on the excited singlet potential energy surface towards a nonplanar cisoid form, followed by internal conversion and rapid attainment of planarity.
\end{abstract}

\section{Introduction}

Spiropyrans can exist in two basic forms: the colourless parent spiropyran structure and an intensely coloured merocyanine structure (fig. 1). The spiropyran structure consists of two orthogonal $\pi$-electron systems with little interaction, so that its absorption spectrum is essentially the superposition of the spectra of the constituent chromophores [1]. Upon excitation of the pyran chromophore in the $\mathrm{UV}$, the bond between the spiro carbon atom and the oxygen atom is broken and the molecule can unfold. The $\pi$ clectrons can cventually conjugate over the cntire structure, so that the corresponding absorption spectrum is displaced far to the red compared to that of the parent form.

The "photochromism" of the spiropyrans has been

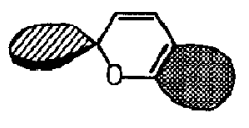

SPIROPYRAN (colourless)

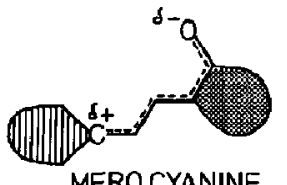

MERO CYANINE various isomers (coloured)
Fig. 1. General scheme for the photochromism of spiropyrans. studied intensively (an excellent review up to 1969 is given in ref. [2] ), not least because of hopes for practical applications. One important class of photochromic spiropyrans is derived from $1^{\prime}, 3^{\prime}, 3^{\prime}$-trimethylspiro-[2H-1-benzopyran-2,2'-indoline ],commonly termed BIPS (cf. fig.2, structure I). Of the derivatives, 6-nitro-BIPS has been examined most thoroughly.

The following topical areas have emerged:

(i) Distinction of merocyanine isomers [3-5], and the photochemical distribution of these isomers;

(ii) Participation of triplet states in the photochcmical reaction of nitro-substituted compounds $[6,7]$;

(iii) Photoinduced aggregation of the coloured structure from 6-nitro-BIPS, mediated through triplet states [8];

(iv) Description of reaction intermediates. The existence of a primary photoproduct in which the $\mathrm{C}_{\text {spiro- }}{ }^{-}$ $O$ bond is broken, and which still possesses the orthogonal parent geometry, was originally proposed in ref. [9]. This hypothetical photoproduct was labelled $\mathrm{X}$; its approximate geometry is shown as structure II in fig. 2. However, its spectral properties, its states and their lifetimes are poorly characterized at present. By definition, $\mathrm{X}$ should be the first photochemical reaction product prior to geometrical 

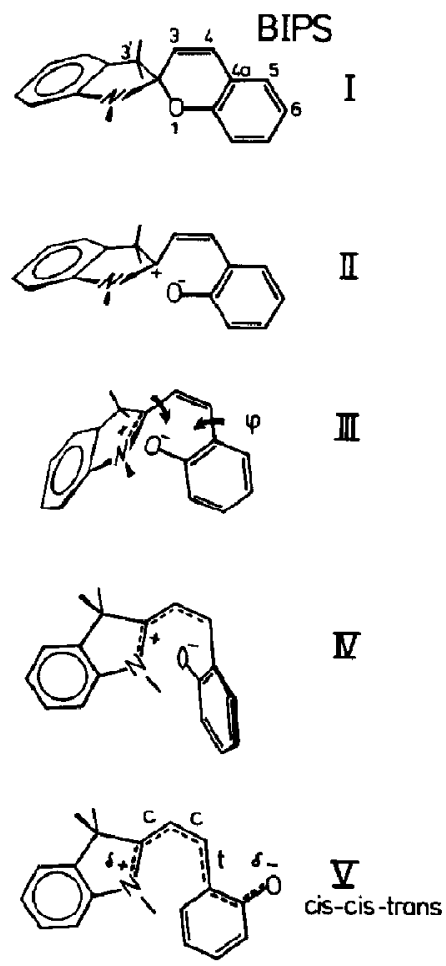

Fig. 2. The structure of BIPS (I), the hypothetical first photochemical reaction product X (II or III), the postulated non-planar cisoid intermediate (IV) and a merocyanine isomer (V). A possible photochemical reaction coordinate $\phi-$ twisting around the $\mathrm{C}_{4}-\mathrm{C}_{4 \mathrm{a}}$ bond - is indicated.

change. Hence the most substantial evidence should come from the earliest spectral data after photolysis. Ref. [10] reported a transient absorption spectrum for 6-nitro-BIPS, taken 10 ps after UV excitation with $6 \mathrm{ps}$ resolution. The spectrum consists of a dominant band centered at $440 \mathrm{~nm}$ and a weaker, red band at $585 \mathrm{~nm}$. Both were attributed to $X$ (in the ground state), although the red band resembles the merocyanine absorption into which the entire spectrum evolves. The decay of $X$ was monitored at $440 \mathrm{~nm}$, giving time constants of 30 and $300 \mathrm{ns.} \mathrm{Other} \mathrm{reports}$ inferred the lifetime of $X$ from the rise of merocyanine absorption, yielding values of 270 ps [8] or 22 ns [11]. Very weak phosphorescence from ${ }^{3} \mathrm{X}$ at 4.2 $K$ was described in ref. [12], while an absorption at $430 \mathrm{~nm}$ with a decay time of $2 \mu$ s at room temperature was also attributed to ${ }^{3} \mathrm{X}[11]$.

The present study attempts to describe the early part of the photochemical conversion of a spiropyran into the corresponding merocyanine isomers. BIPS was chosen as a model compound because its photocoloration proceeds exclusively through singlet states [13]. Pump/probe absorption spectra were recorded at $0.33 \mathrm{ps}$ intervals with $0.42 \mathrm{ps}$ resolution, covering the photochemical reaction from its beginning up to $100 \mathrm{ps}$.

\section{Experimental}

\subsection{The pump/probe absorption experiment}

The pump/probe absorption experiment used a laser system combining an excimer laser with a colliding pulse mode (CPM) dye laser (see below). The pump pulse at $308 \mathrm{~nm}$ (with $360 \mu \mathrm{J}$ focused onto $\approx 3 \mathrm{~mm}^{2}$ ) had a pulse duration of $0.24 \mathrm{ps}$. The probe continuum was generated over $1 \mathrm{~mm}$ of $o$-phosphoric acid [14], from a pulse at $616 \mathrm{~nm}(30 \mu \mathrm{J}$, diffraction limited) of 0.150 ps duration.

The beam of white light was split into the probe beam proper and a reference beam. Both beams were focused onto the sample cuvette; the probe beam was superimposed by the copropagating pump beam. The pump pulse could be advanced in time, relative to the probe pulse, by translation of a mirror on a precision linear actuator. The probe and reference spots were imaged onto separate single quartz fibers which were fed into a spectrograph (Jobin-Yvon H25, 150 lines $/ \mathrm{mm}$ ) using adapting input optics. The probe beam was polarised at the magic angle relative to the pump polarisation by using a sheet polarisor after the sample cuvette. Probe and reference spectra were detected by two photodiode arrays which were intensified by a common multichannel plate (Princeton Instruments). A microcomputer controlled data acquisition and the relative delay between the pump and probe pulses. For each setting of the time delay, 100 separate spectra were recorded at $10 \mathrm{~Hz}$ and averaged numerically.

The temporal resolution is determined by the crosscorrelation width of the pump pulses at $\lambda_{1}=308 \mathrm{~nm}$, with a spectral component at $\lambda_{2}$ of the white probe pulse. The cross-correlation was measured using twophoton absorption in neat benzene (for $\lambda_{2} \leqslant 500 \mathrm{~nm}$ ) or ground-state bleaching of malachite green in 
ethanol (for $\lambda_{2}>500 \mathrm{~nm}$ ). The cross-correlation width (fwhm) was less than 0.42 ps for all probe wavelengths.

An experimental time reference was provided by the peak of the cross-correlation at any probe wavelength $\lambda_{2}$. The cross-correlation peaks occurred simultaneously (within our time resolution) for $\lambda_{2}>430 \mathrm{~nm}$; this is taken as reference time zero. For shorter wavelengths, the peaks were shifted continuously to negative times due to residual dispersive effects: the shift amounted to $-0.33 \mathrm{ps}$ for $\lambda_{2}=395$ $\mathrm{nm}$ and $-0.67 \mathrm{ps}$ for $\lambda_{2}=370 \mathrm{~nm}$. This has to be borne in mind during the discussion of the spectral evolution.

BIPS was synthesized as in ref. [15]. A solution in ethanol $\left(0.40 \times 10^{-2}\right.$ molar $)$ was used in a $0.5 \mathrm{~mm}$ cuvette, giving an optical density of 0.84 at $308 \mathrm{~nm}$.

\subsection{The laser system}

The CPM dye laser has the same design as in ref. [16] and was built from modular components (FSU Jena-Laserinstruments ). It was pumped by $1.1 \mathrm{~W}$ at $514 \mathrm{~nm}$, and produced $100 \mathrm{fs}$ pulses at $616 \mathrm{~nm}$. High stability was achieved through operation in the proper stability regime [17]. The output was amplified [ 18] in modified capillary prismatic dye cells using the first discharge channel of a $\mathrm{XeCl}^{*}$ excimer laser with two channels (Lambda Physik EMG 150). A prism (de-)compressor [19] was used prior to amplification in order to compensate for the effects of group-velocity dispersion. Amplification proceeded along two branches. The first amplified beam was frequency-doubled; the resulting pulse at $308 \mathrm{~nm}$ was further amplified to $1.2 \mathrm{~mJ}$ on a double pass through the second discharge channel of the excimer laser [20]. The second amplified red beam was appropriately delayed for continuum generation. Details will be published elsewhere.

\section{Results}

The transient absorption spectra from an ethanolic solution of BIPS, following excitation at $308 \mathrm{~nm}$ with 0.24 ps pulse duration, are shown in fig. 3 . The spectral development is characterized by the following:
(1) Appearance of a well-defined band at $400 \mathrm{~nm}$ for the earliest times.

(2) Fast evolution (fig. 3a) of this band, to a broad absorption maximising near $440 \mathrm{~nm}$ at 0.33 ps.

(3) From 0.67 ps onward (fig. $3 b$ ), the evolved absorption at $440 \mathrm{~nm}$ decays. During this decay, the broad absorption at longer wavelength increases to form a characteristic band at $550 \mathrm{~nm}$.

The apparent spectral evolution of the primary band in the near $U V$ is a measurement artifact: it may be accounted for by the dependence of time origins on the probe wavelength in this spectral region, as was mentioned in section 2 . The true transient absorption spectrum at a given nominal time may then be constructed from the measured spectrum through substitution of the short-wavelength part by spectrally weighted portions of the two spectra preceding by 0.33 and $0.67 \mathrm{ps}$. In this way, the true absorption spectrum coincident with the pump pulse is seen to cover predominantly the region at wavelengths below $500 \mathrm{~nm}$, with a peak near $440 \mathrm{~nm}$. For later times, the spectral change at shorter wavelengths is relatively small so that this correction is not necessary.

The rise of the absorption at $550 \mathrm{~nm}$, i.e. in the region which is typical for merocyanine absorption, is separately shown in fig. 4. This is compared to the ground-state bleaching of malachite green (in ethanol), which locates the time origin and illustrates the temporal resolution of the measurement. For BIPS, the rise of the absorption at $550 \mathrm{~nm}$ is reasonably well described by a single exponential with a risetime of $1.3 \mathrm{ps}$. A slower, minor component is needed to connect with the optical density at $550 \mathrm{~nm}$ mcasurcd for 104 ps delay (used for normalisation).

\section{Discussion}

The observations above contain a novel feature of spiropyran transient spectroscopy: the immediate appearance of transient absorption in the blue to near UV region only. On the other hand, a component of transient absorption around $450 \mathrm{~nm}$ has been much discussed, although only for 6-nitro-BIPS. In this case it was shown to be dominant at $8 \mathrm{ps}$, and it was assigned to the elusive $\mathrm{X}$ formed in the ground state from a higher triplet of the spiropyran parent [21]. A complication was presented by the simultaneous 

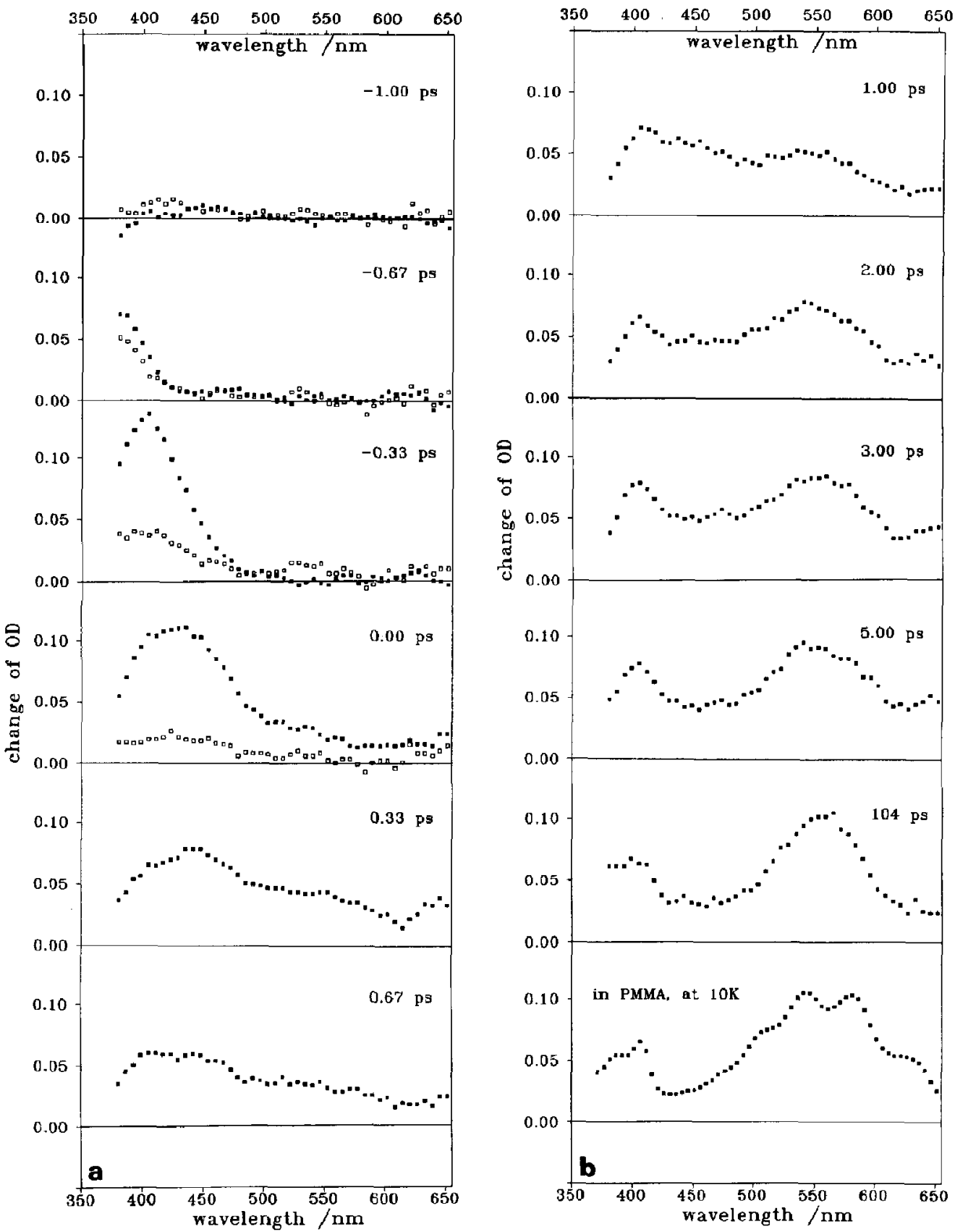

Fig. 3. Transient absorption spectra of BIPS following UV excitation with 0.24 ps pulse duration, and with 0.42 ps resolution. (Open squares indicate induced absorption changes in ethanol alone, due to two-photon absorption of the probe and pump.) 


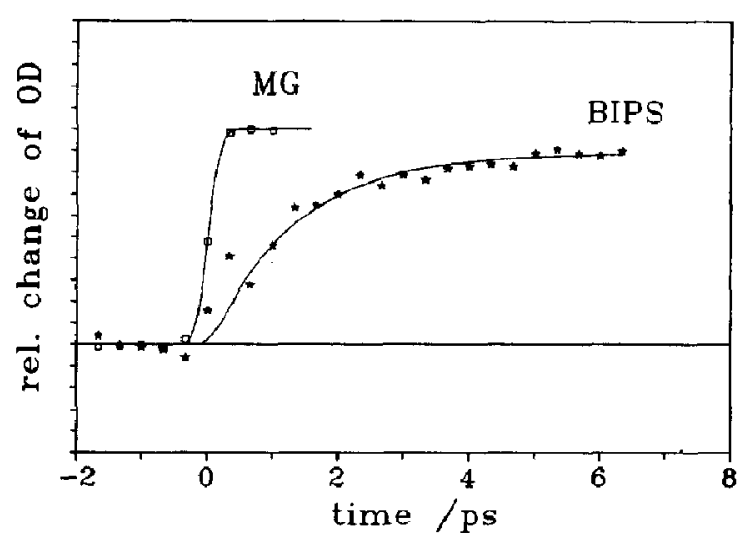

Fig. 4. Rise of transient absorption from an ethanolic solution of BIPS, at $550 \mathrm{~nm}$. This is compared to ground-state bleaching of malachite green (MG) in ethanol. Excitation was at $308 \mathrm{~nm}$ in both cases.

existence of a well-defined red absorption band at this earliest resolved time; this band was also attributed to $X$. Lastly, the $450 \mathrm{~nm}$ region also shows transient absorption due to intermediate triplets [13].

The spectra shown in fig. 3 should not be complicated by intermediate triplets [13]. The spectral evolution distinguishes the $440 \mathrm{~nm}$ absorption from the characteristic red band.

\subsection{Assignments}

The latest recorded transient absorption spectrum, at $104 \mathrm{ps}$ after excitation, strongly resembles the spectrum of the merocyanine products. This is seen by comparison with the stationary merocyanine absorption spectrum shown in the lowest panel of fig. 3b. We therefore assign the transient absorption spectrum at $104 \mathrm{ps}$ to the merocyanine isomer distribution at this time, and in the electronic ground state. Its characteristic absorption band, at $550 \mathrm{~nm}$, was shown to emerge continuously from the broad and unstructured absorption at $0.67 \mathrm{ps,}$, with a risetime of $1.3 \mathrm{ps}$.

At earliest times, up to 0.33 ps after excitation, transient absorption occurs only in the blue to near UV region. This absorption may then have two carriers: a primary photoproduct in its first excited singlet state $S_{1}$, or else a primary photoproduct in its ground state $S_{0}$, which evolves on the $S_{0}$ potential energy surface into some merocyanine isomer distribution. The latter possibility requires that internal conversion take place within 0.3 ps. This does not appear reasonable in view of the wide electronic energy gap. Therefore, we assign the transient absorption around $440 \mathrm{~nm}$, and at earliest times, to an $S_{1-}$ $\mathrm{S}_{n}$ absorption.

It follows that the transient absorption spectra of fig. 3 should record an internal conversion where the spectral evolution from the near UV ends and the merocyanine band appears.

\subsection{Relation to structural change}

What is the likely structural change underlying the spectra? In ref. [22] two paths of spiropyran ring opening were examined by MNDO and INDO-2 calculations for small model systems. The photochemical path was associated with a change of the twist angle $\phi$ around the $\mathrm{C}_{4}-\mathrm{C}_{4 a}$ bond (cf. fig. 2). In the course of this internal rotation, the group attached to the spiro carbon atom rotates into planarity with the central cisoid $\mathrm{C}_{\text {spiro }}-\mathrm{C}_{3}-\mathrm{C}_{4}-\mathrm{C}_{4 \mathrm{a}}$ structure. It will be shown below that our observations are consistent with this description of the photochemical reaction.

Fig. 5 shows potential energy curves for twisting of the $\mathrm{C}_{4}-\mathrm{C}_{4 \mathrm{a}}$ bond in BIPS. These curves are not entirely arbitrary. The spiro form has an $S_{0}-S_{1}$ transition energy of $\approx 3.77 \mathrm{eV}$. The lowest $S_{0}$ reaction enthalpy was determined to be $0.19 \mathrm{eV}$ [3]; the $S_{0}$ reaction enthalpy for an unfavourable CCT isomer (cf. fig. 2) was calculated to be $1.25 \mathrm{eV}$ for chromene [22]; here we assume a reasonable value of 1.0

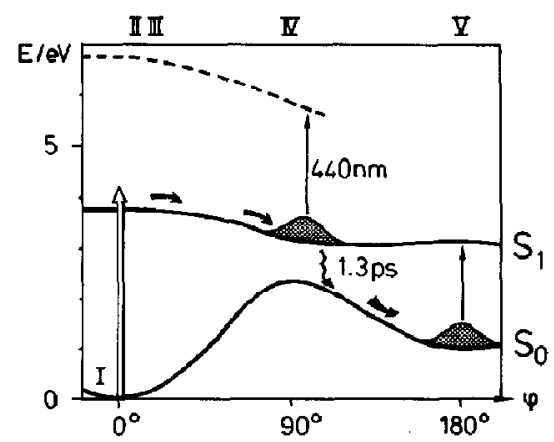

Fig. 5. Description of the photochemical ring-opening reaction along the twist angle $\phi$. Roman numerals refer to structures in fig. 2. 
eV. An $\mathrm{S}_{0}$ barrier of $0.93 \mathrm{eV}$ (in the forward direction) was determined experimentally from the thermal reaction [3]; the calculated value for the photochemical path of chromene is $2.4 \mathrm{eV}$ [22], which is used here. Finally, the $S_{0}-S_{1}$ transition energy for the first merocyanine isomer, $2.2 \mathrm{eV}$, is taken from the transient absorption spectrum at 104 ps.

In combination with the spectral assignments made in section 4.1, we propose the following tentative description of the photochemical ring-opening reaction (figs. 2 and 5 ). Excitation of the spiropyran (I) leads to some primary, transient photoproduct (II or III) on the $S_{1}$ energy surface. For more viscous solvents, it should be detectable by its $S_{1}-S_{n}$ absorption. The photoproduct evolves through barrierless isomerisation on the $S_{1}$ surface to an orthogonal form IV (cf. fig. 2) which is characterized by a broad $S_{1}-S_{n}$ absorption maximising near $440 \mathrm{~nm}$. From here, level crossing to the $S_{0}$ surface occurs through vibronic interaction, with a rate of $0.8 \times 10^{12} \mathrm{~s}^{-1}$. This is followed by faster (and hence unrecorded) ground-state relaxation to the first merocyanine form $\mathbf{V}$.

\section{Conclusion}

In summary we conclude:

(1) There is no evidence for a reasonably stable primary photoproduct $X$ having the parent geometry.

(2) The earliest transient absorption at $440 \mathrm{~nm}$ could be due to a cisoid, non-planar form on the $S_{1}$ surface, representing a local energy minimum which acts as a "funnel" for internal conversion. Internal conversion should be followed by rapid opening to some fully planar first isomer (which need not necessarily have the CCT conformation of structure $\mathrm{V}$ shown in fig. 2).

\section{Acknowledgement}

It is a pleasure to acknowledge the close cooperation with Dr. M. Kaschke (FSU Jena, GDR) in setting up the CPM dye laser and in the initial phase of the amplification work. The author thanks Professor
F.P. Schäfer for his constant interest and a critical reading of the manuscript, and Th. Arthen-Engeland for the low-temperature spectrum. The Deutsche Forschungsgemeinschaft is gratefully acknowledged for support through the Leibniz Prize Program.

\section{References}

[1] N.W. Tyer Jr. and R.S. Becker, J. Am. Chem. Soc. 92 (1970) 1289.

[2] R.C. Bertelson, in: Photochromism, ed. G.H. Brown (Wiley-Interscience, New York, 1971 ) p. 45.

[3] T. Bercovici, R. Heiligman-Rim and E. Fischer, Mol. Photochem. 1 (1969) 23.

[4] C. Balny, P. Douzou, T. Bercovici and E. Fischer, Mol. Photochem. 1 ( 1969$) 225$.

[5] H. Takahashi, K. Yoda, H. Isaka, T. Ohzeki and Y. Sakaino, Chem. Phys. Letters 140 (1987) 90.

[6] T. Beronvici and E. Fischer, J. Am. Chem. Soc. 86 (1964) 5687.

[7] A. Kellmann, L. Lindqvist, S. Monti, F. Tfibel and R. Guglielmetti, J. Photochem. 21 (1983) 223.

[8] Y. Kalisky, T.E. Orlowski and D.J. Williams, J. Phys. Chem. 87 (1983) 5333.

[9] R. Heiligman-Rim, Y. Hirshberg and E. Fischer, J. Phys. Chem. 66 (1962) 2470.

[10] S.A. Krysanov and M.V. Alfimov, Laser Chem. 4 (1984) 129.

[11] C. Lenoble and R.S. Becker, J. Phys. Chem. 90 (1986) 62.

[12] M. Gehrtz, Chr. Brăuchle and J. Voitländer, J. Am. Chem. Soc. 104 (1982) 2094.

[13] C. Lenoble and R.S. Becker, J. Photochem. 34 (1986) 83.

[14] R.R. Alfano and P.P. Ho, IEEE J. Quantum Electron. QE24 (1988) 351.

[15] C.F. Koelsch and W.R. Workman, J. Am. Chem. Soc. 74 (1952) 6288.

[16] J.J. Fontaine, W. Dietel and J.-C. Diels, IEEE J. Quantum Electron. QE-19 (1983) 1467.

[17] E. Döpel, private communication; S. De Silvestri, L. YuPu, V. Magni and O. Svelto, Technical Paper ThBB3 of the European Conference on Quantum Electronics, Hannover (1988).

[18] C. Rolland and P.B. Corkum, Opt. Commun. 59 (1986) 64.

[19] F. Salin and A. Brun, J. Appl. Phys. 61 (1987) 4736.

[20] B. Dick, S. Szatmari, B. Racz and F.P. Schäfer, Opt. Comm. 62 (1987) 277.

[21] S.A. Krysanov and M.V. Alfimov, Chem. Phys. Letters 91 (1982) 77.

[22] F. Zerbetto, S. Monti and G. Orlandi, J. Chem. Soc. Faraday Trans. II 80 (1984) 1513. 